\title{
Structural and molecular analyses of functional epitopes and escape mutants in Japanese encephalitis virus envelope protein domain III
}

\author{
Urmi Roy ${ }^{1}$ (D) \\ Published online: 22 May 2020 \\ (C) Springer Science+Business Media, LLC, part of Springer Nature 2020
}

\begin{abstract}
The Japanese encephalitis virus (JEV) is one of the vector borne causes of encephalitis found in southeastern Asia. This positive single-stranded RNA virus is a member of the Flaviviridae family, which notably includes dengue, tick-borne, West Nile, Zika as well as yellow fever, and transmits to humans by infected mosquitos. The main site of interactions for antibodies against this virus is the envelope protein domain III (ED3). The present report investigates the time-dependent structural and conformational changes of JEV ED3 functional epitopes and escape mutants by computer simulations. The results indicate the presence of significant structural differences between the functional epitopes and the escape mutants. Mutation-induced structural/ conformational instabilities of this type can decrease the antibody neutralization activity. Among the different escape mutants studied here, Ser40Lys/Asp41Arg appear to be most unstable, while Ser40Glu/Asp41Leu exhibit the lowest structural variations. The highest level of escape mutation observed in Ser40Lys is linked to the relatively higher values of root mean square deviation/ fluctuation found in the molecular dynamics simulation of this protein. Secondary-structure deviations and depletion of $\mathrm{H}$ bonding are other contributing factors to the protein's increased instability. Overall, the proteins with residue 41 mutations are found to be structurally more ordered than those with residue 40 mutations. The detailed time-based structural assessment of the mutant epitopes described here may contribute to the development of novel vaccines and antiviral drugs necessary to defend against future outbreaks of JEV escape mutants.
\end{abstract}

Keywords Envelope protein · Escape mutation · Japanese encephalitis virus $\cdot$ Simulation $\cdot$ Structural immunology

\section{Introduction}

Structure-based molecular immunology is an emerging field of biological research, where experimental/clinical immunological results are combined with computer modeling by incorporating bioinformatics tools and commonly available biological/immunological databases [1-6]. Applications of this approach include identifying host/pathogen interactions, predicting epitopes, antibody modeling and designing rational drugs, as well as in silico vaccinations [7-11]. The present work is a computational study within this field, focusing

Electronic supplementary material The online version of this article (https://doi.org/10.1007/s12026-020-09130-y) contains supplementary material, which is available to authorized users.

Urmi Roy

urmi@clarkson.edu

1 Department of Chemistry \& Biomolecular Science, Clarkson University, 8 Clarkson Avenue, Potsdam, NY 13699-5820, USA specifically on certain structural and molecular aspects of the functional epitopes and escape mutants of Japanese encephalitis virus (JEV) envelope protein domain III (ED3).

The JEV is the primary cause for viral encephalitis disease that is responsible for nearly 15,000 deaths annually worldwide, mostly in southeastern Asia [12-14]. Although this disease is predominantly confined within a particular region of the world, after the recent outbreak of several such (previously considered strictly regional) diseases, it is difficult to ignore the potential global impact of any spreadable illness. JEV is a vector borne disease that transmits to humans via cycles between infected Culex mosquitos and vertebrates. The singlestranded RNA positive JEV belongs to the Flaviviridae family that also includes dengue, tick-borne encephalitis, West Nile, Zika, and yellow fever viruses. The flavivirus consists of three structural portions: (i) capsid, commonly known as C; (ii) premembrane or membrane protein, PrM or M; and (iii) envelope protein $\mathrm{E}$. The $\mathrm{E}$ protein (a homodimer) is considered to be the main site for host-virus attachment and consists of three structural domains: domain 1 (D1), domain II (D2), and domain III 
(D3). The envelope protein D3 (ED3) is the main interacting site for the JEV neutralizing antibodies. The non-structural (NS) protein includes seven nonstructural units [15-21].

The NMR and X-ray crystal structures of ED3 for West Nile, tick-borne Langat, yellow fever, and different dengue virus serotypes have already been archived in the protein databank (PDB). Likewise, the crystal structure of the complete envelope protein of JEV is also available in the literature [21], and the structure of the corresponding ED3 has been identified as 1PJW.PDB [22]. In view of the scope for preventive and therapeutic interventions, the significance of the ED3 epitopes and "neutralization escape" mutants of ED3 in the Flaviviridae family has been noted in several earlier studies [15-17, 20, 23, 24]. Previous authors have also identified certain regions/residues on the JEV-E protein as determining factors for functional epitopes [21, 22, 25-30]. While experimental research about the Flaviviridae virus family has been active for a number of years, molecular level structural/ computational studies of conformational changes (involving functional epitopes and escape mutants) of the JEV ED3 have so far remained comparatively less explored.

Specifically, residues Ser331 and Asp332 on ED3 of JEV (strain: Beijing-1) are believed to interact with corresponding residues of $\mathrm{H} 3$ region in monoclonal antibody $(\mathrm{mAb}) \mathrm{E} 3.3$ [27]. Alterations of Ser331 and Asp332 on ED3 can significantly lower their binding affinity toward specific $\mathrm{mAb}$ sites, and therefore, these critical residue mutations behave like "neutralizing antibody" escapes. By using site-directed mutagenesis and ELISA affinity assay, Lin and $\mathrm{Wu}$ have shown that, the altered 331 and 332 residues, (Ser331Lys, Ser331Arg, and Ser331Glu) and (Asp332Leu, Asp332Lys, and Asp332Arg) in JEV ED3 fusion proteins undergo complete loss of binding affinity against mAb E3.3. However, there are four additional variants (Ser331Leu, Ser331Gln/ Asp332Gln, Asp332Glu) and Ala substitutions at position 331 and 332 that exhibit moderate to low reductions in their binding affinities toward $\mathrm{mAb}$ E3.3. The reasons why these residue mutations would cause a decrease or a complete loss of function (neutralizing activity) have also been discussed previously [27]. This present work centers on the impact of escape mutants on the structure and function of the overall ED3.

Molecular dynamics (MD) simulation $[31,32]$ is used here to characterize the time-dependent molecular level structural changes of both wild type (wt) and mutant JEV ED3 proteins in the solution phase. MD simulation is an established technique, useful for identifying structure-function relationships of proteins in general. Previous MD-based studies by the present author have described the structures and time-dependent dynamics of several immunologically relevant proteins [33-38]. Other authors have reported MD simulation studies of the dengue ED3 protein in the aqueous medium $[39,40]$. The primary goal of the current work is to investigate the time- dependent structural changes of some of the neutralizing escape mutant proteins as those described by Lin and $\mathrm{Wu}$ on the JEV ED3 [27]. The motivation for this effort stems from the observation that such residue level structural studies using wt or mutant JEV ED3 proteins may provide useful information in epitope mapping and novel antibody-based therapeutics.

\section{Materials and methods}

The solution structure of D3 of the JEV envelope protein 1PJW.PDB has been described by Wu et al. [22]. 1PJW is a single chain protein with 111 amino acids. For the purpose of MD simulation, wt JEV ED3, 1PJW (chain A; model 1), and its structural variants, each with single residue mutation, are used [27]. The simulations have used Nanoscale Molecular Dynamics (NAMD) [41] and Visual Molecular Dynamics (VMD) [42] programs.

JEV ED3 residues 40 and 41 of 1PJW correspond to residues 331 and 332 of the 3P54, JEV E protein shown in Figs. 1 and 2 and Figs. S1-S2 of the Electronic supplementary material (ESM). Mutations at position 40 and 41 were induced using the mutator plugin of the VMD. Mutator is a built in feature of VMD that changes the residue of interest using internal coordinates. Six sets of mutant protein structures were generated, each carrying a single point mutation at position 40 or 41. The data for these six mutated proteins are presented in Figs. 3, 4 and 5, along with further results included in Figs. S3-S4 of the ESM. Four additional variants, with two sets for each position, are also shown in the ESM, Figs. S5-S7.

The test system was set up with explicit solvation while the counter ions, sodium or chloride, were added as needed to neutralize the system. First, using the NPT ensemble, each system was energy-minimized and equilibrated for 10,000 steps. The final production run was performed for $5 \mathrm{~ns}$ using the NVT ensemble at a constant temperature of 298 K. The CHARMM force field was used. Periodic boundary conditions and Langevin dynamics were applied for each of the systems studied. The particle mesh Ewald (PME) method was activated for each run. The integration time step was $1.0 \mathrm{fs}$, and the total number of steps during the final run was set to $5 \times 10^{6}$, which corresponded to $5 \mathrm{~ns}$ in length. MD simulations of such time scale can be carried out with relatively moderate computational resources and, at the same time, can serve as an effective probe of the basic conformational changes in the protein $[43,44]$. The detailed output frequencies of the trajectory coordinate files have been described previously [33-36]. The VMD program [42] was used to analyze these trajectory structures, and the corresponding figures were generated with Discovery Studio Visualizer (DSV) [45]. Origin 9.1 was used for trajectory analyses. 
Fig. 1 Structural analyses of JEV E protein. a Schematic of JEV structure. Seven non-structural parts (NS1, NS2a, NS2b, NS3, NS4a, NS4b, and NS5) along with protein's structural components $(\mathrm{C}, \mathrm{M}$, and $\mathrm{E})$ are displayed. b Secondary structure of of JEV E protein 3P54.PDB where ED3 is displayed in green and ED3 residues Ser331 and Asp332 are shown in CPK mode. $\mathbf{c}$ Residues at inter-domain interfaces. Interfacial residues that are close to D3 are depicted in gray line mode a

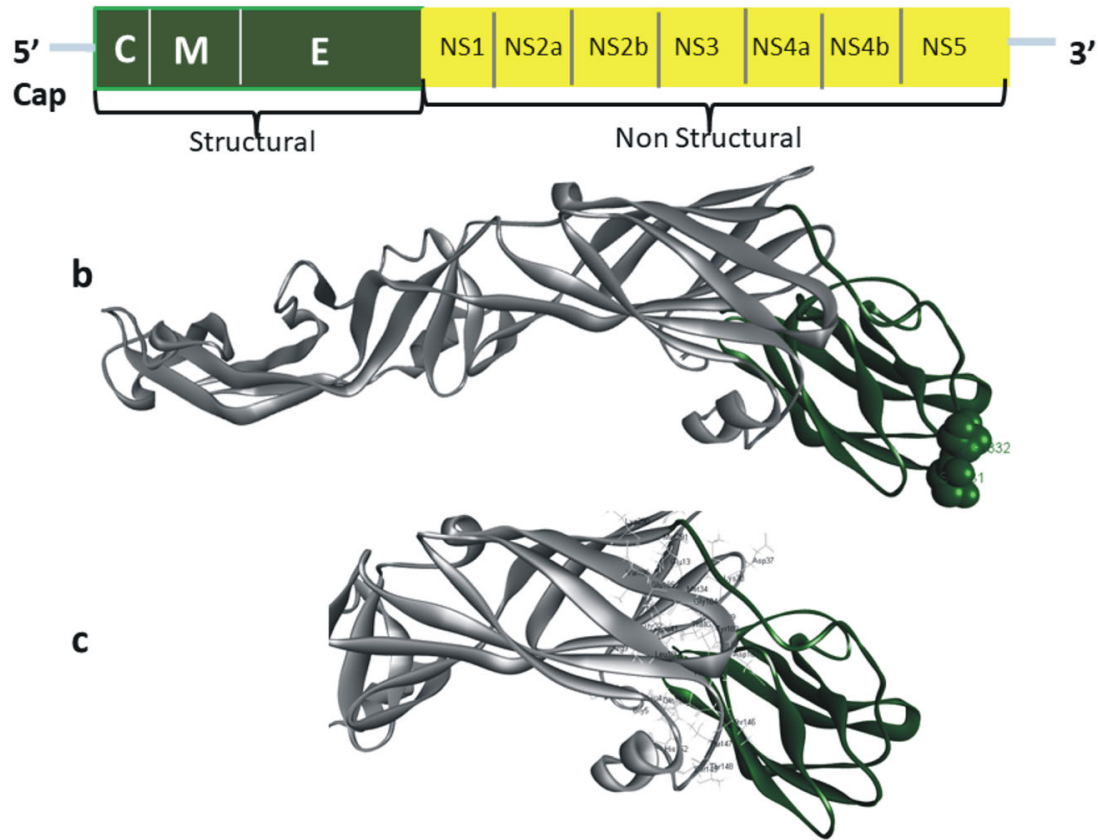

\section{Results}

In general, epitopes play a key role in molecular recognition and antibody-antigen interactions. Certain epitope mutations may lead to a lack of antibody neutralization or, may demonstrate high neutralizing effectiveness. For the JEV envelope protein, majority of these neutralizing agents are located at the terminal end of the ED3. Some of these JEV ED3 escape mutants have been described by Wu et al. [22]. In addition, as shown by Luca and co-authors, the E3 and E3.3 antibodies can recognize several residues (Gly302, Ile337, Phe360 and Arg387) on the JEV ED3 lateral boundary [21]. According to
Lin and $\mathrm{Wu}$, various residues of the envelope proteins $\mathrm{D} 1$ and D2 may also take part in the binding process of $\mathrm{mAb}$ E3.3 to ED3 [27]. As part of this present study, the structures of wt JEV ED3 protein [22] and its corresponding mutant variants are compared [27], and time-based structural changes of these JEV ED3 wt and mutant proteins are identified.

Figure 1 shows the various structural features of JEV ED3. To supplement these results, the amino acid sequence alignments between 3P54 and 1PJW have been included in the ESM, Fig. S1. Different neutralization escape mutations of JEV ED3 have been tabulated in ESM Fig. S2. Figure 1a shows a schematic view of the JEV structure. A ribbon

Fig. 2 Structure of JEV ED3 protein. a Secondary structure of 1PJW.PDB, where Ser40 and Asp41 are displayed in stick mode. $\mathbf{b}, \mathbf{c}$ Neighbor residues of Ser40 and Asp41 in 1PJW. d Solvent accessible surface of 1PJW. e Surface representation of 1PJW based on hydrophobicity

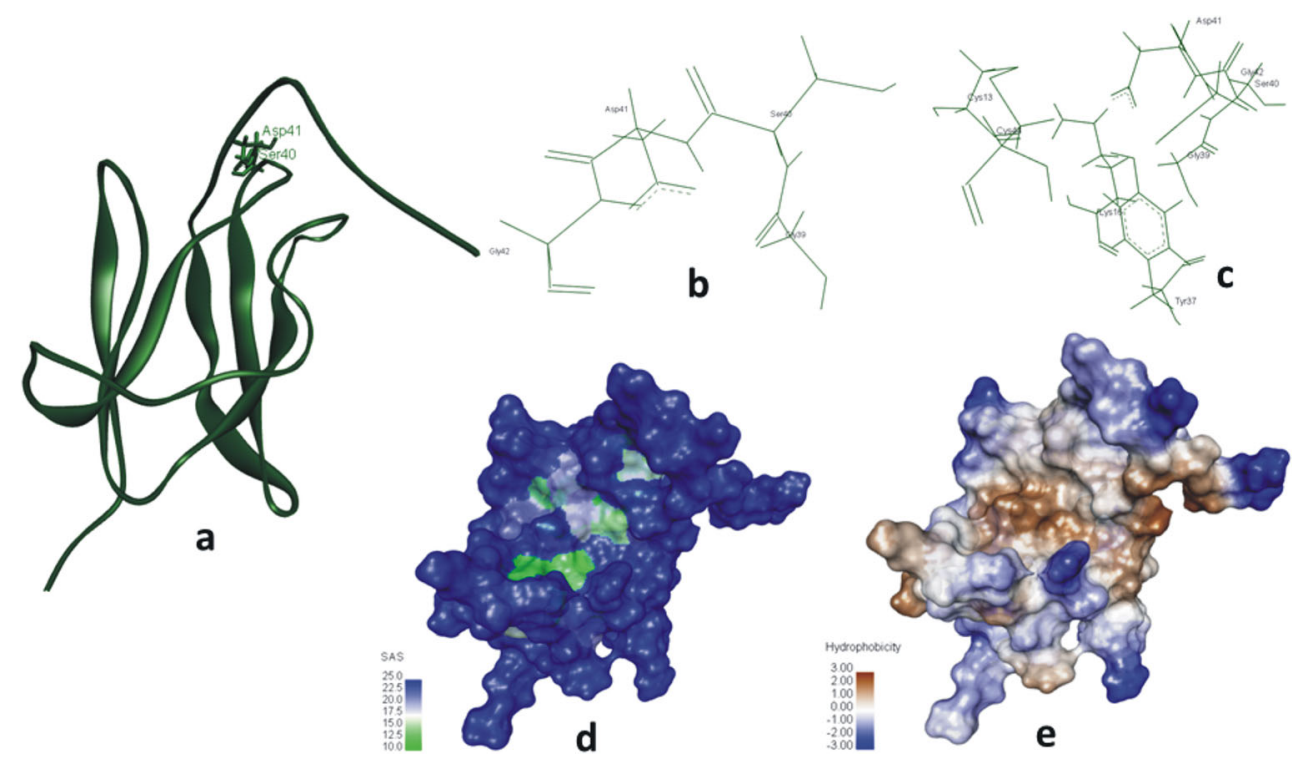



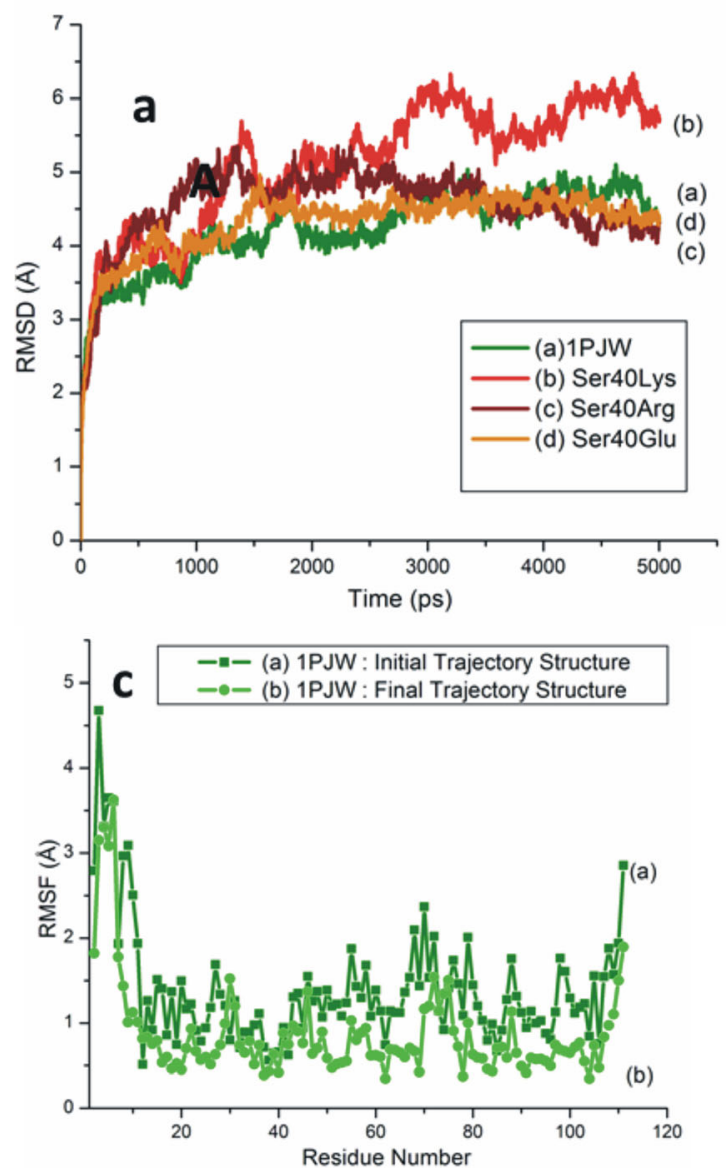

Fig. 3 MD simulations of wt and mutant versions of JEV ED3. a RMSD comparison of wt ED3 protein and its three mutant versions each with single residue point mutation at position 40 (Ser40Lys, Ser40Arg and Ser40Glu). b RMSD data for wt (Ser) and mutant residues (Lys, Arg

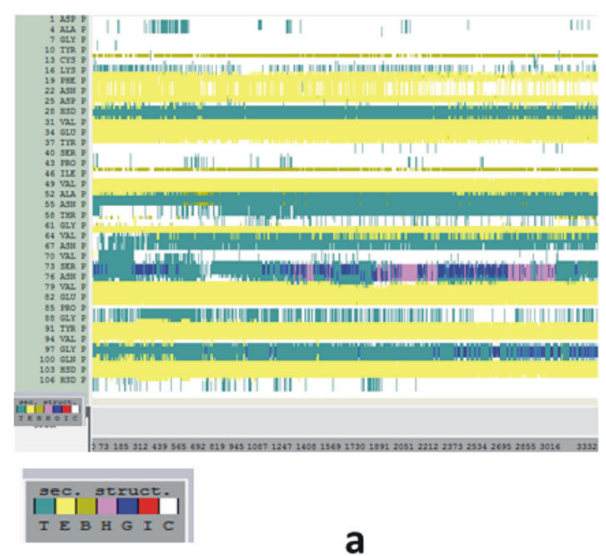

Fig. 4 a Protein's secondary structure changes during 5 ns MD simulation. Illustration of the conformational changes in 1PJW.PDB during the timescale of the MD simulation. Color code explanation of the secondary strucures are shown in the small panel below (a). T denotes turn (aqua); E represents the $\beta$-sheet (yellow); B is the representative of isolated bridges (dark yellow); $\mathrm{H}$ indicates $\alpha$-helix (pink); $\mathrm{G}$ is the $3-10$

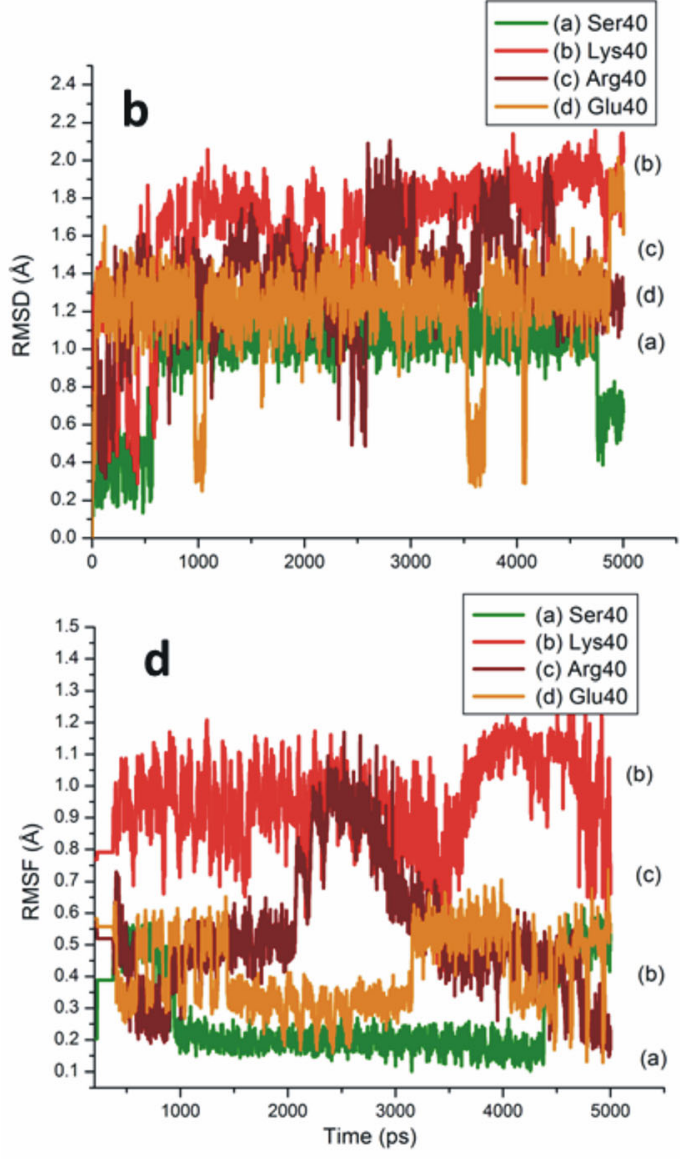

and Glu) at position 40 during the $5 \mathrm{~ns}$ time scale. All-atom RMSD values are considered in a and $\mathbf{b}$. $\mathbf{c}$ RMSF analyses of 1PJW. The overlay of $\mathbf{a}$ initial and $\mathbf{b}$ final conformations are diaplayed. $\mathbf{d}$ RMSF analyses of wt residue Ser40 and mutant residues (Lys, Arg and Glu) at position 40

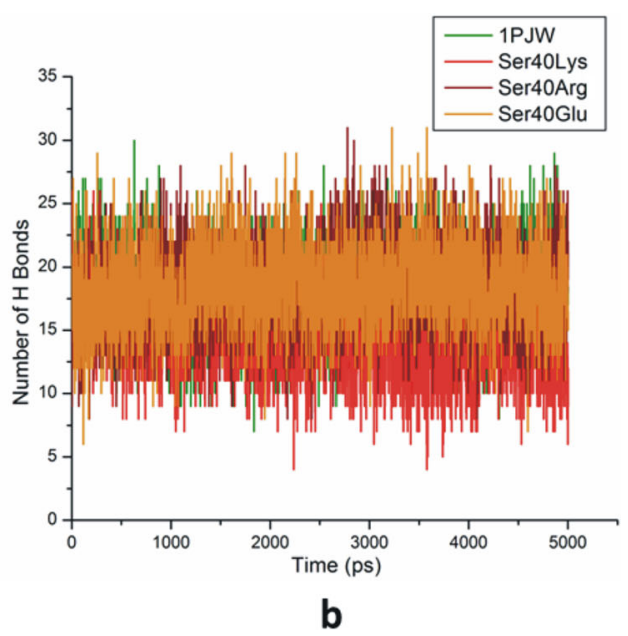

helix (blue); pi helix, I is colored in red and coils, C are colored in white. These temporal structural analyses are created using VMD timeline plugin window. Default color codes are used in this picture. $\mathbf{b} \mathrm{H}$ bond formation of wt and mutant structures (each protein with single residue mutation at position 40) of JEV ED3 during the time course of MD simulation 

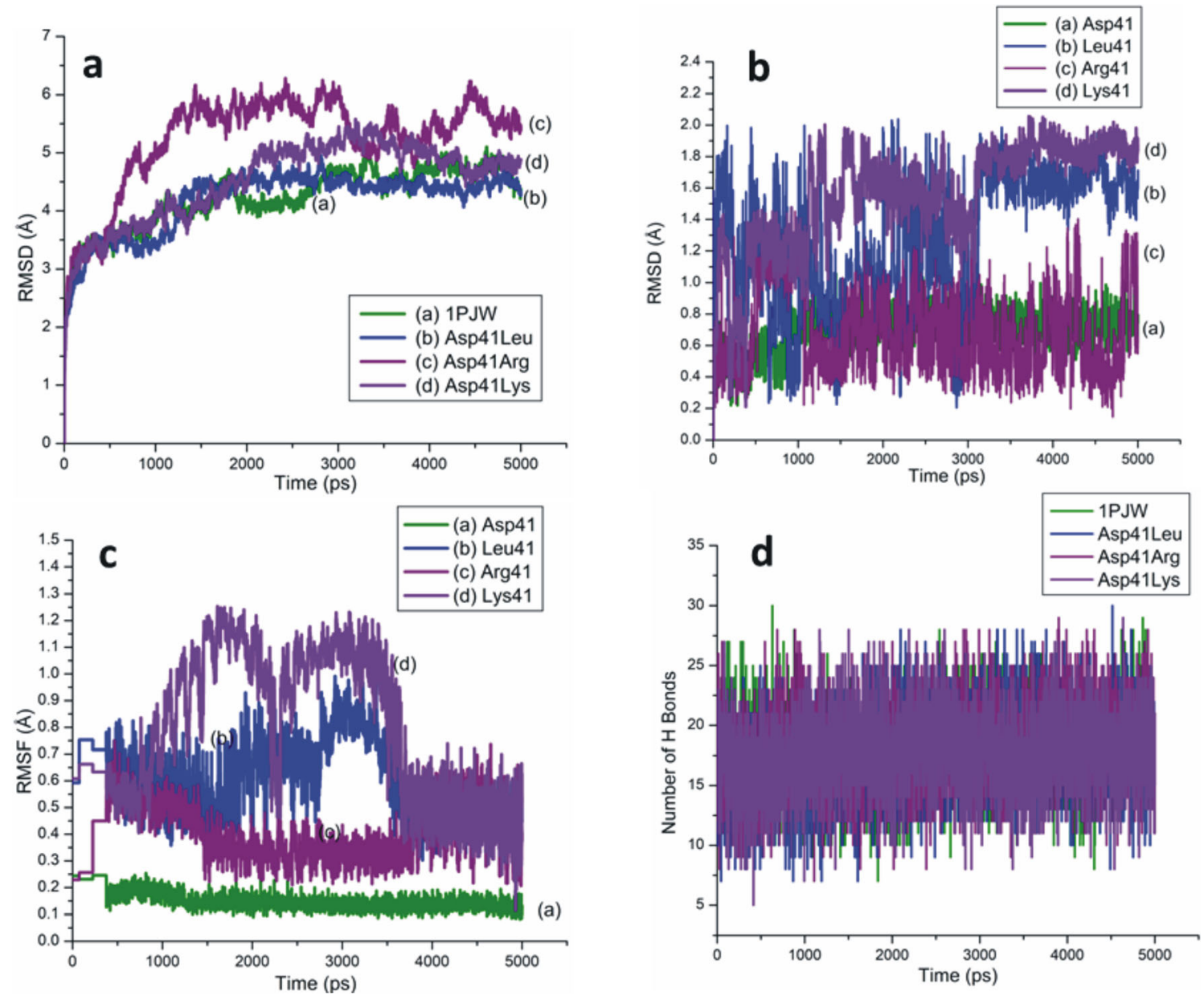

Fig. 5 MD simulations of wt and mutant forms of JEV ED3. a Comparison of RMSD plots between wt ED3 protein and its three mutant forms each with single point mutations (Asp41Leu, Asp41Arg and Asp41Lys) during the timescale of MD simulation. b The RMSD analyses of the wt (Asp) and mutant residues (Leu, Arg and Lys) at

diagram of JEV E protein (3P54.PDB) is displayed in Fig. 1b where ED3 is indicated in green. The residues at position 331 and 332 are shown in Fig. 1b. The interfacial residues that exist in close vicinity of D3 are depicted in Fig. 1c.

The positions of two functional "antigenic determinant" residues, Ser40 and Asp41 in wt JEV ED3 1PJW, are displayed in Fig. 2a. The locations of these residues are marked on the Figure, and as seen here, the two labeled residues are positioned next to each other at the exterior surface of the protein. The neighboring residues of Ser40 and Asp41 in 1PJW are displayed in Fig. $2 b$ and c, where the relevant sites are once again marked. The surface illustrations, based on solvent accessibility and hydrophobicity, are presented in Fig. $2 \mathrm{~d}$ and e, where the associated color intensity codes indicate, respectively, the solvent accessible surfaces (SAS) and the hydrophobicity indices (with more positive values for higher hydrophobicities). A close examination of the surface plots reveals that the wt residues are mostly solvent accessible with larger SAS indices. However, in a separate examination,

position 41 during the simulation time scale. All-atom RMSD values are considered in all these cases. $\mathbf{c}$ RMSF plots of wt and mutant residues at position 41 during MD simulation. $\mathbf{d ~} \mathrm{H}$ bond formation of wt protein and its three mutant forms (each with single amino acid substitution at position 41) during the time course of MD simulation

it was found that the mutant Lys40 and Arg41 had more solvent accessible surfaces than those of the wt residues (data not included here). As will be discussed later in this report, this has implications in terms of stability for antibody binding.

Structural variations of the mutant proteins were investigated by MD simulations. Results of these simulations for wt JEV ED3 and the mutant variants (residue mutation at position 40) are shown in Fig. 3a. Within the framework of MD simulation, root mean square deviation (RMSD) calculations measure the time-dependent structural variations in biomolecular systems. Among the three mutant proteins considered here, the mutation of Ser40Lys (plot b) in Fig. 3a shows the highest RMSD values compared with those of the (a) wt 1PJW and the other two structural variants, (c) Ser40Arg and (d) Ser40Glu. The mutant proteins Ser40Arg and Ser40Glu have slightly lower RMSD values than those of the wt protein (Fig 3a), especially during the last $1 \mathrm{~ns}$ of the simulation. Mostly, similar results are observed also for the individual mutant residues Arg40 and Glu40 (Fig. 3b). The 
mutant Lys40 residue indicates slightly higher RMSD values than those of wt Ser40 and the other two mutant variants at position 40. Per residue root mean square fluctuation (RMSF) analyses of wt 1PJW are considered in Fig. 3c, which displays mutual overlays of the initial and final conformations as functions of residue numbers. Figure $3 d$ presents the RMSF temporal profile analyses of (a) wt residue Ser40, along with those of three mutant residues (b) Lys, (c) Arg, and (d) Glu at position 40. Overall, the observed RMSF graphs of wt protein, 1PJW, and wt residue Ser40 are almost uniform in the native structures, compared with those of the three mutants.

Figure 4a shows secondary structural changes of 1PJW during the simulation time. The horizontal axis represents frame number of simulation, and the vertical axis lists the amino acid residues of the protein. Structural variations are noticeable around residues 70-76, which explain the somewhat higher RMSF values observed around that region (Fig. $3 \mathrm{c}$ ). The counts of $\mathrm{H}$ bond formation in the wt and mutant JEV ED3 structures (each with single residue mutation at position 40) are displayed in Fig. 4b. The $\mathrm{H}$ bond counts for the Ser40Lys appear with slightly lower values than those of the two mutants and the wt protein. This lowered population of $\mathrm{H}$ bonds in Ser40Lys likely contributes to a corresponding lowering of the protein's structural constancy.

Comparative RMSD plots of wt JEV ED3 and its mutant variants (residue mutation at position 41) are shown in Fig. 5a. According to these plots, Asp41Arg and Asp41Leu, within the comparison group, are associated with the highest and lowest structural variations, respectively. Figure 5b shows the results of RMSD analyses for the three mutant residues at position 41 (Leu, Arg, and Lys); the RMSD plot of wt residue Asp41 is also included for structural comparisons. The RMSF values of all four residues at position 41 are noted in Fig. 5c. In all cases, the wt species (either as a PDB structure, 1PJW, or as an individual residue, ASP41) demonstrate almost uniform RMSD/RMSF variations during the simulation time scale. The $\mathrm{H}$ bond formation counts for the wt and mutant JEV ED3 structures (each with a single residue mutation at position 41) are displayed in Fig. 5d.

To gain further insight in the molecular level structural variations, the changes in the mutant proteins' secondary structure, occurring during the 5 ns timescale, were recorded. These results are shown in ESM, Fig. S3-A-F, where the secondary structure in the loop region around residues $41-45$ and 70-76 shows maximum changeability in the mutant protein Se40Lys. The results of RMSF analyses for the mutant JEV ED3 protein complexes, each with single residue substitution at positions 40 and 41, are presented, respectively, in Fig. S4A-C and S4-D-F of the ESM. In the latter data, higher variations are observed in Asp41Arg, whereas the overall RMSF values remain nearly stable in the wt species, 1PJW. Additional four variants, two for each position (Ser40Leu,
Ser40Gln/ Asp41Gln, Asp41Glu), are also displayed in the ESM, Figs. S5-7.

\section{Discussions}

The wt JEV ED3, 1PJW, has a $\beta$-barrel shaped structure and contains a centrally located buried region with a hydrophobic core (Fig. 2). In flavivirus, ED3 contributes to host-virus interactions and membrane fusion, and JEV ED3 participates in antibody neutralizing actions. Due to these reasons, understanding the structural details of ED3 is particularly relevant to characterize the protein's functions. As noted by previous authors, certain ED3 mutations can lead to the lack of antibody neutralization [15-17, 20, 21, 23, 24, 27]. Some of these JEV ED3 neutralization escape mutations are discussed in the ESM, where the escape mutations are listed in Fig. S2; the structural changes induced by the particular mutations are also discussed there in the context of that Figure.

Among the different escape mutants studied here, Ser40Lys appears to be most unstable, while SerGlu40 exhibited the lowest structural variations. However, the RMSD plot for the wt type is largely time invariant in its overall profile as compared with those of the three mutants (Fig. 3a). The RMSD values of the singular mutant residue Glu40 exhibits are found to be comparable to those of the wt Ser40 residue (Fig. 3b). The moderately stable RMSD values observed for Glu40 can be associated with the latter's negative charge (a relatively higher affinity to bind with hydrogen).

The temporal RMSF profile basically monitors the steadiness of a given protein complex. The RMSF values of initial and final snapshots of wt 1PJW did not vary significantly, and only slightly higher fluctuations are observed near the residue 70 and the terminal regions (Fig. 3c). Residues 70-76 reside within the loop region, and this possibly accounts for the slightly higher structural changeability of the system around this region. The mutant residue Lys40 shows higher fluctuations than those of the wt residue Ser40 and mutant residues Arg40 and Glu40 (Fig. 3d). Once again, here the overall observed RMSF values for the wt type of residue 40 shows minimal variations within the time scale probed here. In comparison, the fluctuations monitored for Lys40 are relatively larger, most probably as a result of the residue's charged nature.

Illustrations of the secondary conformational changes in 1PJW.PDB during the timescale of the MD simulation are presented in Fig. 4a. In wt JEV ED3, the conformational variations (turn to $\alpha$-helix and 3-10 helix) are primarily observed within the loop regions; otherwise, the structural uniformity is fairly maintained. A comparable situation, involving a high flexibility of the protein's certain loop areas, has been previously reported for dengue virus serotype 2 , where the observation could be linked to the virus-host binding process [40]. 
A similar implication for cell binding may apply to the present case.

Although the number of $\mathrm{H}$ bond formation during $\mathrm{MD}$ simulation time is almost steady for the wt and mutant variants of JEV ED3 (Fig. 4b), the slightly lower value in Ser40Lys indicates that it is less stable than the wt as well as the other two mutant variants at position 40. Accordingly, the highest level of escape mutation may be possible in Ser40Lys due to this protein's higher RMSD/RMSF rate, secondary-structure deviation, and loss of $\mathrm{H}$ bonding. A recent study on influenza virus mutant epitope analysis indicates that certain loss of noncovalent interactions in mutant protein may be related to the latter's omission of affinity toward antibody [46]. The authors of that work also suggested that a reduced number of $\mathrm{H}$ bonds in mutant-antigen/Ab complex might be related to weaker antibody attachment. These observations are consistent with the expected role of the mutations for generating antibody neutralizing escape mutants.

From Fig. 5a, it is evident that, among the three mutant proteins, Asp41Arg mutation shows higher RMSD values. Nevertheless, during the last $1 \mathrm{~ns}$ of MD simulation, the wt 1PJW and its other two structural variants, Asp41Leu, Asp41Lys, exhibit RMSDs of comparable magnitudes. Moreover, Asp41Arg have higher RMSD values during the simulation than those of the wt protein; this indicates that a residue substitution most likely does not play a role in stabilizing the protein structure. The protein Asp41Lys also shows considerable RMSD fluctuations (Fig. 5a, b). It is possible that the positive charge of these two residues (Arg and Lys) affects the proteins' stability through an electrostatic mechanism.

While JEV ED3 protein with Asp41Arg mutation exhibits relatively higher variations, the individual mutant residues Leu41 and Lys41 show slightly higher RMSD values than that of the mutant residue Arg41 (Fig. 5b). However, in all cases, the wt species demonstrate a virtually unvarying RMSD profile during the simulation time scale. Robust molecular interactions within the wt protein structure are most likely responsible for the relatively lower and temporally uniform RMSD values observed. At the same time, it is also possible that the mAb does not have a favorable binding conformation for the mutant proteins of unstable conformations.

The above analyses are based on the all-atom RMSD values, computed using initial structure (time $=0 \mathrm{~ns}$ ) as a reference. The results in Fig. 5c indicate that, during the last $1 \mathrm{~ns}$ of simulation, all these three mutant residues at position 41 (Leu, Arg, and Lys) have almost similar RMSF values. Here also, the wt protein shows rather low structural variance. The results in Fig $5 \mathrm{~d}$ suggests that, in terms of $\mathrm{H}$ bonds formation, the wt and mutant JEV ED3 structures (each with single residue mutation at position 41) do not significantly deviate from each other.

The conformational changes of the mutant proteins with an altered residue at position 40 or 41 are examined in Fig. S3A-F. Based on the secondary structure changes, it is possible to infer that, the protein with Ser40Lys is less stable than the wt as well as the other two mutant variants at position 40 (Fig. S3A). Overall proteins, with residue 41 mutations, seem to be more orderly than proteins with residue 40 mutations. The results of RMSF analyses for the mutant JEV ED3 proteins are presented in the ESM, Fig. S4-A-C and S4-D-F, respectively. The protein with Ser40Glu mutation appears most steady (Figs. 3a and Fig. $\mathrm{S} 4 \mathrm{C}$ ) among the first three cases examined. Among the mutant protein with residue substitution at position 41, the higher variations are observed in Asp41Arg, whereas the overall RMSF values almost uniform in wt species, 1PJW (Figs. S4E and 3c).

On the basis of the above discussion, it is reasonable to conclude that Ser40Lys and Asp41Arg are structurally less stable compare to other mutated proteins residing at positions 40 and 41. All the other variants noted above (except for Ser40Lys and Asp41Arg) have a slightly lower or equal RMSD value with respect to that of the wt $1 \mathrm{PJW}$. In particular, during the last phase of the simulation time, though the wt 1PJW has maintained an overall fairly stable status. All these mutant proteins show complete loss of binding toward mAb E3.3 [27].

In West Nile virus, the exterior surface region residues participate extensively in antibody bindings [18]. Therefore, the instability of the mutant epitopes in this region may disrupt the normal course of antibody bindings. It is possible that the greater structural movements of mutant epitopes in JEV ED3 (Figs.3a, d and 5a, c) could be related to their structural instability and may reduce their binding interactions toward $\mathrm{mAb}$. Solvent accessibility may play a role in proteins destabilization. Ieong et al. have shown that, in the influenza virus, mutation-mediated increase of solvent accessibility plays a role in disrupting the salt bridges and, eventually destabilizes the protein [46]. Since the mutant residues Lys40 and Arg41 contain relatively larger solvent accessible surfaces, this accessibility may evidently contribute to the unstable nature of Ser40Lys and Asp41Arg. All other mutant residues at position 40 and 41 also display greater SAS values than those of the wt residues Ser40 and Asp41. Residue mutations at position 40 show greater SAS than mutations at position 41. Finally, the higher RMSD/RMSF rates certainly have an impact on the proteins' overall stability; this may lower the mAb binding probability and finally assist them to be recognized as antibody neutralization escape mutations. In addition to the results presented above, MD simulation data for four more mutant proteins were also obtained. These latter results, presented in ESM Fig. S5A and S5C, demonstrated somewhat higher RMSD values compared with that of the wt 1PJW. These four additional mutations have been known to exhibit decreased binding affinity toward mAb E3.3 [27].

The JEV ED3-specific antibody neutralization is a subject with considerable scopes for further investigations. In this context, the observation reported by Chiou et al. is noted according to which, some mutations on ED1-ED3 of the JEV virus-like particles should not exhibit decreased cross-reactivity toward the 
target $\mathrm{mAbs}$ [47]. Plans for extending this work in the future include trial simulations of model systems based on the $\mathrm{mAb}$ bound JEV ED3 complex to check the neutralizing efficacy of the mutant epitopes. It will also be necessary eventually to probe how the functional epitopes and the escape mutants on ED3 might affect the overall functionality of the ED3-mAb complex. Such predictive modeling efforts could be useful for studying structure-based novel drug targets.

\section{Conclusions}

Epitope-immune responses are crucial for understanding various physiological and biological phenomena, and structural immunology plays a key role in identifying such possible functional epitopes and escape mutants [48-51]. The structures of the functional epitopes and neutralization escape mutants of JEV ED3 have been studied in this work. A major finding of the study is the identification of significant structural differences between the functional epitopes and escape mutants. Such mutation-generated structural and conformational instabilities of proteins can act to alter their functionalities. Among the different escape mutants studied here, Ser40Lys/Asp41Arg are most unstable while SerGlu40/ Asp41Leu are associated with the lowest structural variations. The relatively high RMSD/RMSF values found in the MD results for Ser40Lys can be taken as an indicator of this protein's elevated level of escape mutation, the changes in its secondary-structure, as well as its lower capacity for $\mathrm{H}$ bonding. Overall, the proteins with residue 41 mutations seem more structured than those with residue 40 mutations.

The detailed time-based simulation analyses and structural assessment of the escape mutants as those described in this paper may be helpful for scientists to develop new vaccines and antiviral drugs to combat future outbreaks of JEV antibody neutralization escape mutants. Such characterizations of neutralizing "epitope determinants" on JEV ED3 may be useful in the studies of alternative epitope determinants and new epitope based drug targets and biologics [52]. Additionally, this study may also be useful in aiding the understanding of the rather complex epitope-immune responses.

The importance of investigating viruses has been further recognized most recently, as COVID-19 pandemic has already taken nearly 200,000 human lives worldwide (as of April 27, 2020) [53]. Based on the current observations, the antibody neutralization escape mutants of JEV could potentially become a future threat to public health on a broad scale. Since RNA viruses have greater adaptability and mutation rates, effective antiviral treatments tend to be rather challenging in such cases. Molecular and structural details of epitope mutations can help to address these challenges. These illustrative results of computer simulation presented in this report serve to reiterate this point in the specific context of JEV escape mutants.

Acknowledgments The author acknowledges utilization of the following simulation and visualization software packages: (1) NAMD and (2) VMD: NAMD and VMD, developed by the Theoretical and Computational Biophysics Group in the Beckman Institute for Advanced Science and Technology at the University of Illinois, Urbana-Champaign; (3) Discovery Studio Visualizer: Dassault Systèmes BIOVIA, Discovery Studio Modeling Environment, San Diego, CA: Dassault Systèmes (2015).

\section{Compliance with ethical standards}

Conflict of interest The author declares that she has no conflict of interest.

\section{References}

1. Korber B, LaBute M, Yusim K. Immunoinformatics comes of age. PLoS Comput Biol. 2006;2:e71.

2. Zaslavsky E, Hayot F, Sealfon SC. Computational approaches to understanding dendritic cell responses to influenza virus infection. Immunol Res. 2012;54:160-8.

3. Loyola PKR, Campos-Rodríguez R, Bello M, Rojas-Hernández S, Zimic M, Quiliano M, et al. Theoretical analysis of the neuraminidase epitope of the Mexican A H1N1 influenza strain, and experimental studies on its interaction with rabbit and human hosts. Immunol Res. 2013;56:44-60.

4. Borrego F, Kabat J, Kim DK, Lieto L, Maasho K, Peña J, et al. Structure and function of major histocompatibility complex (MHC) class I specific receptors expressed on human natural killer (NK) cells. Mol Immunol. 2002;38:637-60.

5. Ligoudistianou $\mathrm{C}, \mathrm{Xu} \mathrm{Y}$, Garnier G, Circolo A, Volanakis JE. A novel human complement-related protein, $\mathrm{C} 1 \mathrm{r}$-like protease $(\mathrm{C} 1 \mathrm{r}-$ LP), specifically cleaves pro-C1s. Biochem J. 2005;387:165-73.

6. Kaya T, Swamy N, Persons KS, Ray S, Mohr SC, Ray R. Covalent labeling of nuclear vitamin $\mathrm{D}$ receptor with affinity labeling reagents containing a cross-linking probe at three different positions of the parent ligand: structural and biochemical implications. Bioorg Chem. 2009;37:57-63.

7. Li Y, Llewellyn L, Moczydlowski E. Biochemical and immunochemical comparison of saxiphilin and transferrin, two structurally related plasma proteins from Rana catesbeiana. Mol Pharmacol. 1993;44:742-8.

8. Cai Z, Yarovoi SV, Zhu Z, Rauova L, Hayes V, Lebedeva T, et al. Atomic description of the immune complex involved in heparininduced thrombocytopenia. Nat Commun. 2015;6:8277.

9. Luck LA, Mason AB, Savage KJ, MacGillivray RTA, Woodworth RC. 19F NMR studies of the recombinant human transferrin N-lobe and three single point mutants. Magn Reson Chem. 1997;35:477-81.

10. Dube S, Abbott L, Abbott L, Dube DK, Dolcini G, Gutierrez S, et al. The complete genomic sequence of an in vivo low replicating BLV strain. Virol J. 2009;6:120.

11. Bazzone LE, King M, MacKay CR, Kyawe PP, Meraner P, Lindstrom D, Rojas-Quintero J, Owen CA, Wang JP, Brass AL, Kurt-Jones EA, Finberg RW. A disintegrin and metalloproteinase 9 domain (ADAM9) Is a major susceptibility Factor in the early stages of encephalomyocarditis virus infection. mBio. 2019;10.

12. Erlanger TE, Weiss S, Keiser J, Utzinger J, Wiedenmayer K. Past, present, and future of Japanese encephalitis. Emerg Infect Dis. 2009;15:1-7. 
13. Misra UK, Kalita J. Overview: Japanese encephalitis. Prog Neurobiol. 2010;91:108-20.

14. Ghosh D, Basu A. Japanese encephalitis-a pathological and clinical perspective. PLoS Negl Trop Dis. 2009;3:e437-e.

15. Rey FA, Heinz FX, Mandl C, Kunz C, Harrison SC. The envelope glycoprotein from tick-borne encephalitis virus at 2 A resolution. Nature. 1995;375:291-8.

16. Crill WD, Roehrig JT. Monoclonal antibodies that bind to domain III of dengue virus $\mathrm{E}$ glycoprotein are the most efficient blockers of virus adsorption to Vero cells. J Virol. 2001;75:7769-73.

17. Beasley DW, Barrett AD. Identification of neutralizing epitopes within structural domain III of the West Nile virus envelope protein. J Virol. 2002;76:13097-100.

18. Volk DE, Beasley DWC, Kallick DA, Holbrook MR, Barrett ADT, Gorenstein DG. Solution structure and antibody binding studies of the envelope protein domain III from the New York strain of West Nile virus. J Biol Chem. 2004;279:38755-61.

19. Mukhopadhyay S, Kuhn RJ, Rossmann MG. A structural perspective of the flavivirus life cycle. Nat Rev Microbiol. 2005;3:13-22.

20. Kanai R, Kar K, Anthony K, Gould LH, Ledizet M, Fikrig E, et al. Crystal structure of West Nile virus envelope glycoprotein reveals viral surface epitopes. J Virol. 2006;80:11000-8.

21. Luca VC, AbiMansour J, Nelson CA, Fremont DH. Crystal structure of the Japanese encephalitis virus envelope protein. J Virol. 2012;86:2337-46.

22. Wu K-P, Wu C-W, Tsao Y-P, Kuo T-W, Lou Y-C, Lin C-W, et al. Structural basis of a Flavivirus recognized by its neutralizing antibody: solution structure of the domain III of the Japanese encephalitis virus envelope protein. J Biol Chem. 2003;278:46007-13.

23. Hiramatsu K, Tadano M, Men R, Lai CJ. Mutational analysis of a neutralization epitope on the dengue type 2 virus (DEN2) envelope protein: monoclonal antibody resistant DEN2/DEN4 chimeras exhibit reduced mouse neurovirulence. Virology. 1996;224:437-45.

24. Crill WD, Chang GJ. Localization and characterization of flavivirus envelope glycoprotein cross-reactive epitopes. J Virol. 2004;78: 13975-86.

25. Wu S-C, Lian W-C, Hsu L-C, Liau M-Y. Japanese encephalitis virus antigenic variants with characteristic differences in neutralization resistance and mouse virulence. Virus Res. 1997;51:173-81.

26. Wu S-C, Lin $\mathrm{C}-\mathrm{W}$. Neutralizing peptide ligands selected from phage-displayed libraries mimic the conformational epitope on domain III of the Japanese encephalitis virus envelope protein. Virus Res. 2001;76:59-69.

27. Lin $\mathrm{C}-\mathrm{W}, \mathrm{Wu} \mathrm{S}-\mathrm{C}$. A functional epitope determinant on domain III of the Japanese encephalitis virus envelope protein interacted with neutralizing-antibody combining sites. J Virol. 2003;77:2600-6.

28. Hasegawa H, Yoshida M, Shiosaka T, Fujita S, Kobayashi Y. Mutations in the envelope protein of Japanese encephalitis virus affect entry into cultured cells and virulence in mice. Virology. 1992;191:158-65.

29. Seif SA, Morita K, Matsuo S, Hasebe F, Igarashi A. Finer mapping of neutralizing epitope(s) on the C-terminal of Japanese encephalitis virus E-protein expressed in recombinant Escherichia coli system. Vaccine. 1995; 13:1515-21.

30. Kolaskar AS, Kulkarni-Kale U. Prediction of three-dimensional structure and mapping of conformational epitopes of envelope glycoprotein of Japanese encephalitis virus. Virology. 1999;261:31-42.

31. Durrant JD, McCammon JA. Molecular dynamics simulations and drug discovery. BMC Biol. 2011;9:71.

32. Sheinerman FB, Brooks CL 3rd. A molecular dynamics simulation study of segment B1 of protein G. Proteins. 1997;29:193-202.

33. Roy U. Structural characterizations of the Fas receptor and the Fasassociated protein with death domain interactions. Protein J. 2016;35:51-60.
34. Roy U. Structural modeling of tumor necrosis factor: a protein of immunological importance. Biotechnol Appl Biochem. 2017;64: 454-63.

35. Roy U. Structure and function of an inflammatory cytokine, interleukin-2, analyzed using the bioinformatic approach. Protein J. 2019;38:525-36.

36. Roy U. 3D modeling of tumor necrosis factor receptor and tumor necrosis factor-bound receptor systems. Mol Informatics. 2019;38: 1800011.

37. Roy U. Structural biology of tumor necrosis factor demonstrated for undergraduate instruction by computer simulation. Biochem Mol Biol Educ. 2016;44:246-55.

38. Roy U, Luck LA. Molecular modeling of estrogen receptor using molecular operating environment. Biochem Mol Biol Educ. 2007;35:238-43.

39. Dubey KD, Chaubey AK, Ojha RP. Role of $\mathrm{pH}$ on dimeric interactions for DENV envelope protein: an insight from molecular dynamics study. Biochim Biophys Acta. 1814;2011:1796-801.

40. Soares ROS, Caliri A. Stereochemical features of the envelope protein domain III of dengue virus reveals putative antigenic site in the five-fold symmetry axis. Biochim Biophys Acta Proteins Proteomics. 2013;1834:221-30.

41. Phillips JC, Braun R, Wang W, Gumbart J, Tajkhorshid E, Villa E, et al. Scalable molecular dynamics with NAMD. J Comput Chem. 2005;26:1781-802.

42. Humphrey W, Dalke A, Schulten K. VMD: visual molecular dynamics. J Mol Graph. 1996;14:33-8.

43. Shen M, Zhou S, Li Y, Pan P, Zhang L, Hou T. Discovery and optimization of triazine derivatives as ROCK1 inhibitors: molecular docking, molecular dynamics simulations and free energy calculations. Mol BioSyst. 2013;9:361-74.

44. Sun H, Jiang YJ, Yu QS, Luo CC, Zou JW. Effect of mutation K85R on GSK-3 $\beta$ : molecular dynamics simulation. Biochem Biophys Res Commun. 2008:377:962-5.

45. Dassault Systèmes BIOVIA. Discovery studio modeling environment. San Diego: Dassault Systèmes; 2015.

46. Ieong P, Amaro RE, Li WW. Molecular dynamics analysis of antibody recognition and escape by human H1N1 influenza hemagglutinin. Biophys J. 2015;108:2704-12.

47. Chiou SS, Fan YC, Crill WD, Chang RY, Chang GJ. Mutation analysis of the cross-reactive epitopes of Japanese encephalitis virus envelope glycoprotein. J Gen Virol. 2012;93:1185-92.

48. Greenspan NS, Di Cera E. Defining epitopes: it's not as easy as it seems. Nat Biotech. 1999;17:936-7.

49. Ndifon W, Wingreen NS, Levin SA. Differential neutralization efficiency of hemagglutinin epitopes, antibody interference, and the design of influenza vaccines. Proc Natl Acad Sci. 2009;106:8701-6.

50. Mason AB, Byrne SL, Everse SJ, Roberts SE, Chasteen ND, Smith $\mathrm{VC}$, et al. A loop in the N-lobe of human serum transferrin is critical for binding to the transferrin receptor as revealed by mutagenesis, isothermal titration calorimetry, and epitope mapping. J Mol Recognit. 2009;22:521-9.

51. Dilioglou S, Cruse JM, Lewis RE. High panel reactive antibody against cross-reactive group antigens as a contraindication to renal allotransplantation. Exp Mol Pathol. 2001;71:73-8.

52. Wise MC, Xu Z, Tello-Ruiz E, Beck C, Trautz A, Patel A, et al. In vivo delivery of synthetic DNA-encoded antibodies induces broad HIV-1-neutralizing activity. J Clin Invest. 2020;130:827-37.

53. WHO report-98. In: COVID-2019 situation reports. https://www. who.int/emergencies/diseases/novel-coronavirus-2019/situationreports/. Accessed 27 April 2020

Publisher's note Springer Nature remains neutral with regard to jurisdictional claims in published maps and institutional affiliations. 